\title{
MANAGEMENT OF UNUSUALLY FUSED MAXILLARY CENTRAL INCISOR: CASE REPORT
}

\author{
Fahd A. Aljarbou*
}

\begin{abstract}
The management of fused maxillary central incisor can be difficult. This study presents a treated fused maxillary central incisor done in a single visit. Cone beam computed tomography (CBCT) was very helpful to overview the anatomy of this tooth and plan it prior to treatment. Supernumerary part was removed, and double access of each coronal parts were done separately allowing non-surgical root canal treatment of the two roots followed by final composite resin restoration. The patient was referred back to his orthodontist to continue the planned treatment. The patient was recalled and the overall outcome was successful.
\end{abstract}

KEYWORDS: CBCT, Fused tooth, Multidisciplinary, Unusual tooth, Supernumerary tooth

\section{INTRODUCTION}

Dental anatomy is a wide topic that studies teeth at microstructure and macrostructure levels ${ }^{(1)}$. Normally, there would be eight teeth in each quadrant, but that number might change because of tooth extraction or the presence of dental anomalies. These anomalies can occur at early stages of teeth development such as tooth fusion or gemination. A glossary of endodontic terminology defines tooth fusion as "A 'double' tooth resulting from the union of two adjacent tooth germs" while tooth gemination is "A disturbance during odontogenesis in which partial cleavage of the tooth germ occurs and results in a tooth that has a double or 'twin' crown; usually not completely separated; common root and pulp space shared" (2). Clearly from the definition, the clinical similarity between the previously mentioned teeth anomalies is high and differentiation is challenging. It will be more challenging if further dental anomalies were encountered at the same time, such as having supernumerary teeth. It can affect both dentitions with low prevalence varying according to ethnicity in which it ranges from $5 \%$ in Japanese to $0.5 \%$ in Caucasians ${ }^{(3)}$. Even the management of such anomaly is variable ranging from doing nothing if it affects primary teeth to a multidisciplinary approach in the case of permanent dentition ${ }^{(4,5)}$.

The main objective of any treatment starts by addressing the patient's chief complaint. Most of the

\footnotetext{
* Associate Professor, Department of Restorative Dental Sciences, Division of Endodontics, College of Dentistry,
} King Saud University, Riyadh, Saudi Arabia 
complaints that are associated with teeth anomalies include higher caries potential, more periodontal diseases, poor aesthetics and dental malocclusion ${ }^{(6)}$. This case report aims to present the management of an unusually fused maxillary central incisor planned using cone-beam computed tomography (CBCT).

\section{Case report:}

An 18-year-old man of Arabic descent, with no contributing medical condition contradicting any dental treatment, was referred from an orthodontist. $\mathrm{He}$ requested our plan from an endodontic perspective, but he mandated removing the interfering supernumerary tooth to permit the proper occlusal adjustment in the area of the incisors. Proximal stripping was also planned and tried but the patient felt pain. The patient complained of tooth hypersensitivity to cold drinks recently and poor aesthetics for 12 years. Initial screening of the case was done as shown in Fig. 1. Extraoral and intraoral examination was within normal limits. Endodontic examination of the adjacent teeth was normal to a cold test, electric pulp test, percussion and palpation tests but the offending tooth revealed sharp response to a cold test that lasted less than five seconds. Probing depth was within $2-3 \mathrm{~mm}$ on the buccal and palatal aspects and bleeding was noted around the supernumerary tooth indicating simple gingivitis. A carious lesion around the supernumerary tooth was also observed. After a thorough case discussion, elective nonsurgical root canal treatment was planned for both fused teeth as two separate accesses combined with the removal of the supernumerary tooth. Informed consent was granted for the treatment and case reporting.

One carpule of $1.8 \mathrm{~mL}$ solution of lidocaine $2 \%$ with 1:80,000 epinephrine (Lidocaine $\mathrm{HCl}$, Huons Co., Seoul, Korea) was used for buccal and palatal infiltration anesthesia of tooth \#11. Rubber dam was used to isolate the operating field and OpalDam (Ultradent Products, Inc., South Jordan, UT, USA) was used to optimize the isolation. Drilling the fused supernumerary tooth was established using a long shank round-ended tapered diamond bur followed by two separate accesses at $45^{\circ}$ angle using the same bur (toward the two canals). No. $2 \& 3$ Gates Glidden drills (Union Broach Co., Long Island City, NY) were used to widen the orifices and the access to the isthmus area between the two roots. Electronic determination of working length was performed using a size $15 \mathrm{~K}$-hand file (Dentsply Co., Munich, Germany) from the incisal edges. The working length was $22 \mathrm{~mm}$ for both roots. Rotary instrumentation was performed up to size 35 , using the entire sequence of ProTaper ${ }^{\circledR}$ Gold (Dentsply Tulsa Dental Specialties, Tulsa, OK, USA) up to size F3, followed by ProFile Ni-Ti files (size: 35, 0.04 taper) (Maillefer, Ballaigues, Switzerland). Irrigation was performed with $12 \mathrm{cc}$ of $5.25 \%$ sodium hypochlorite between each file and as a final rinse after using $5 \mathrm{ml}$ of 17\% EDTA (Meta Biomed Co. Ltd., Mandaluyong, Korea). The size 15 K-hand file was curved to facilitate pulp removal at the isthmus area and aided by sonic agitation using an EndoActivator System (Dentsply Tulsa Dental Specialties, Tulsa, OK). Then, the canal was dried with sterile paper points and obturated with the single-cone technique using an Endosequence BC sealer (Brasseler USA, Savannah, GA) and guttapercha cones (size: 35, 0.04 taper) (Meta Biomed Co. Ltd., Cheongju City, Chungbuk, Korea). The palatal access openings were sealed using MultiCore Flow (Ivoclar Vivadent, Schaan, Liechtenstein). Fig. 1 presents the preoperative assessment including radiographic findings and clinical photos Fig. 2 presents an overview assessment using CBCT. Fig. 3 presents the radiographic steps of the nonsurgical root canal treatment of the fused root.

The patient was reassessed a week later, and he was asymptomatic. Six months follow up just before the extraction of the fused tooth revealed normal findings. 

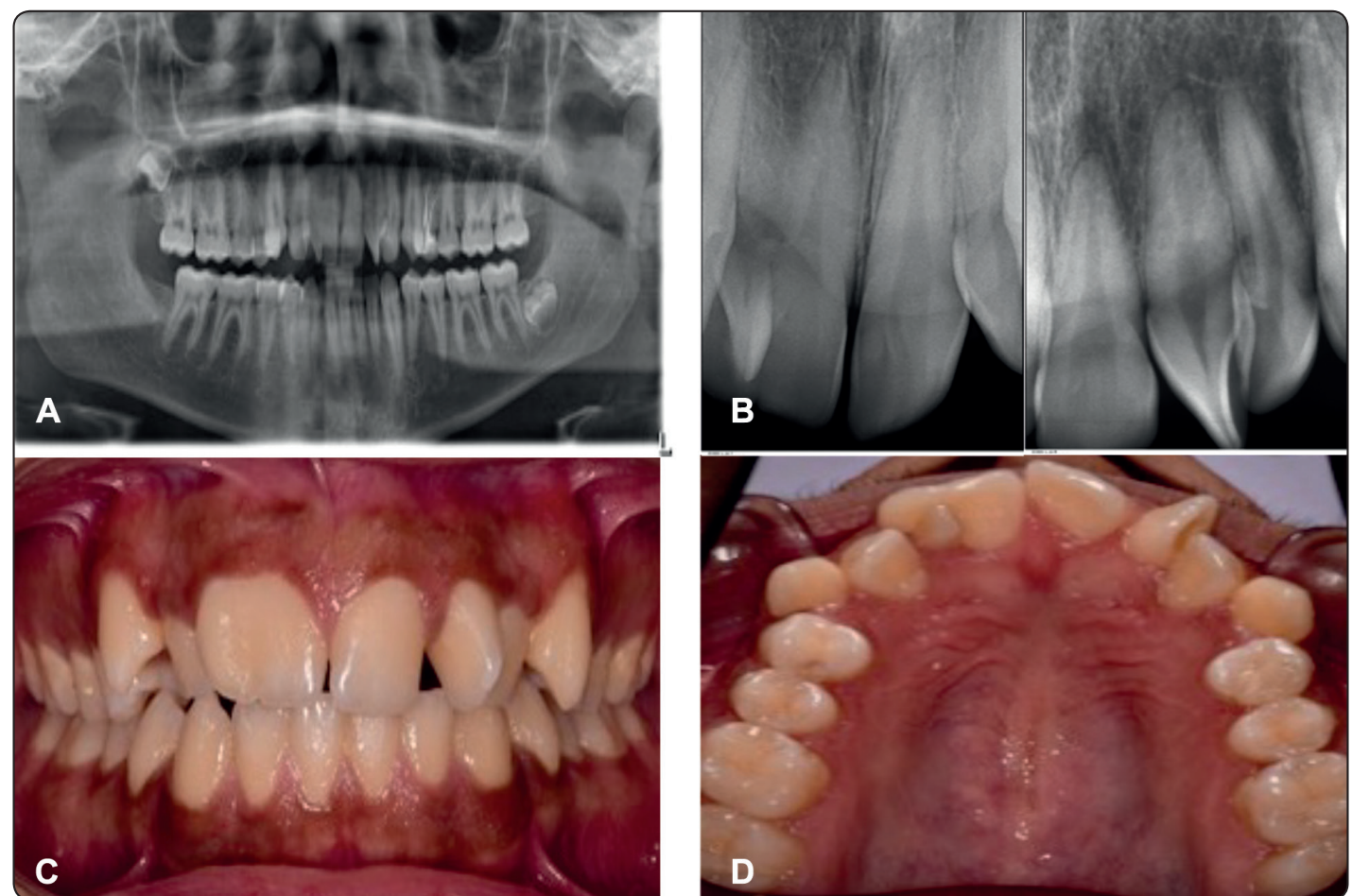

Fig. (1) Preoperative radiographic and clinical screening of the fused root; A) Panoramic radiograph presenting the overall patient dentition. B) two periapical radiographs of the central incisors at both quadrants, the preoperative radiograph of the fused central incisor \#11 has an overlapping with an evidence of narrow pulpal space. C) Frontal view (buccal view) at the maximum intercuspation position. D) Upper occlusal picture showing the palatal aspect of the fused root.
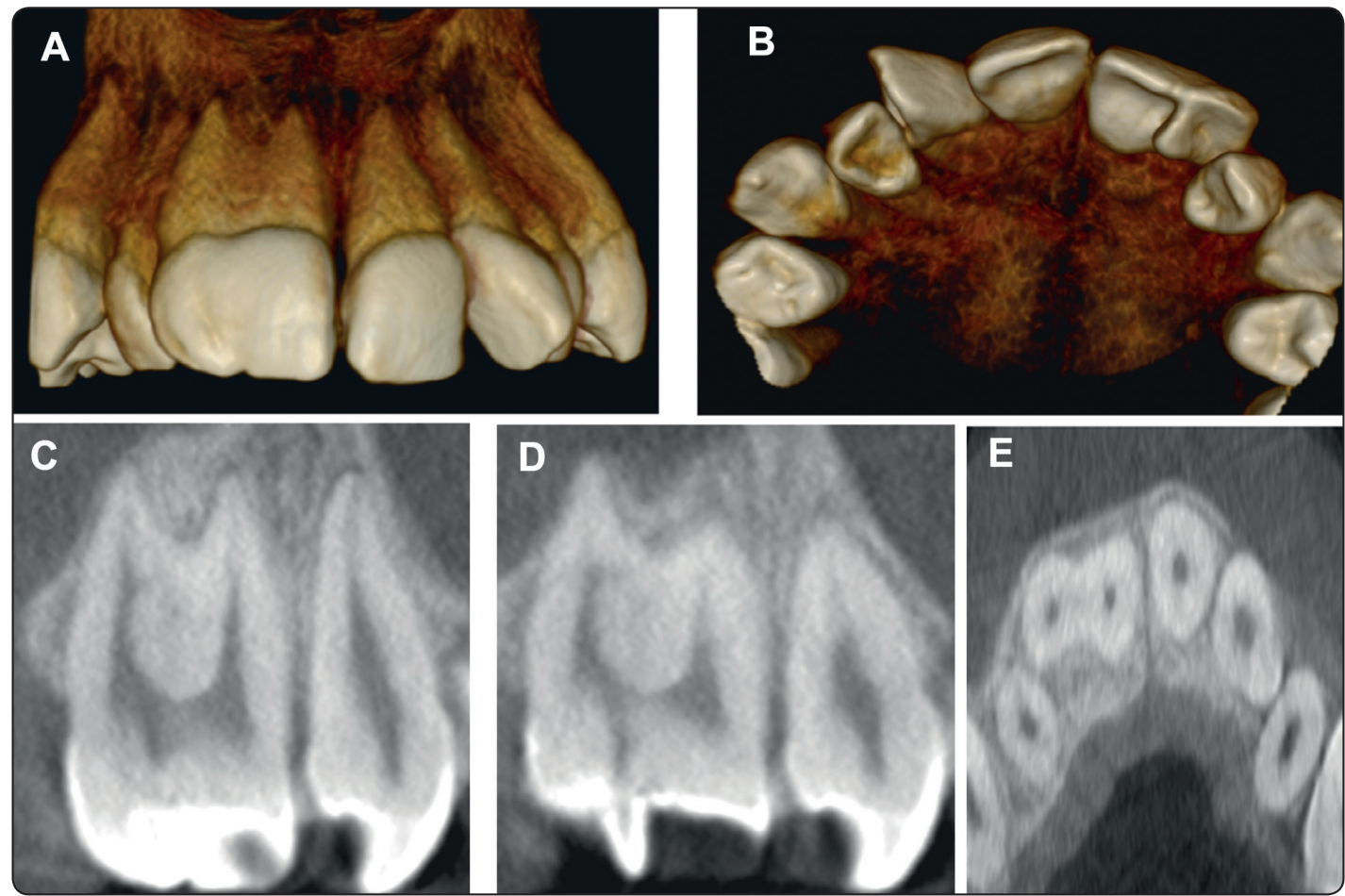

Fig. (2) Overview from Cone beam computed tomography scans; A- Buccal view from a 3D construction of tooth \#11. B- Palatal view from a 3D construction. C- Coronal section for the fused root. D- Coronal section illustrating the pulpal extension towards the supernumerary tooth. E- Axial section of tooth \#11 at mid root. 


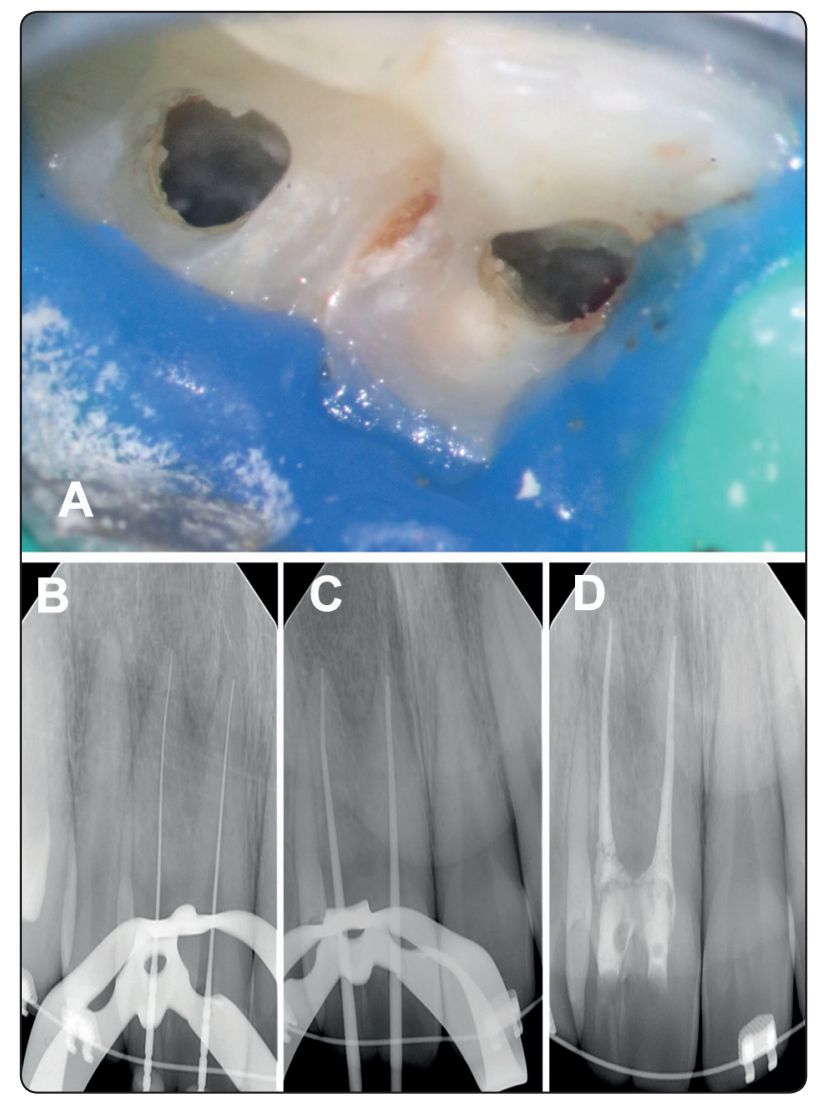

Fig. (3) Steps of the non-surgical root canal treatment of the fused root; A- Access cavities for both roots with an evidence of interconnection between the two pulp chambers. Supernumerary tooth was removed by a bur. B. Working length radiograph with size $15 \mathrm{~K}$-files. C. Cone fit radiograph showing size 35 guttapercha cones. D. Final radiograph.

\section{DISCUSSION}

The patient's main complaint was poor aesthetics. He was told by a general dentist when he was six years old that no treatment should be rendered before he reaches 18 years old. This suggestion led to further complications including malocclusion and premature contact on the supernumerary tooth, gingival inflammation and overgrowth palatally associated with the simple carious lesion. Recently, he was evaluated by an orthodontist who suggested extraction of the fused tooth and mesialize tooth \#21 to the space of tooth \#11 then mesialize the extra tooth that holds the morphological characteristics of upper central incisor in the second quadrant towards the space of tooth \#21. This proposed treatment option was refused by the patient due to aesthetic reasons. Alternatively, a plan to remove the interfering fused supernumerary tooth combined with pulpal removal to control the patient's sensitivity and allows orthodontic stripping while mesialization is in progress. This multidisciplinary treatment could have been avoided if the case had been properly addressed by an orthodontist who might have started orthodontic treatment at an earlier stage.

CBCT was critical in treatment planning. It is recommended to use it in teeth with unusual anatomy ${ }^{(7)}$. As shown in the figures, the fused tooth shared both the crown and more than two-thirds of the root while the pulpal connection was at the level of the pulp chamber with a narrow pulpal extension toward the supernumerary part. Knowing this was made possible with the help of CBCT.

The endodontic treatment option selected was elective nonsurgical root canal treatment. Removal of the supernumerary and the carious lesion together might approach the pulp and worsen patient symptoms. The pulpotomy option was discussed but because pulpal space is shared in the chamber, it would be difficult to control the materials in that area without affecting the radicular part. Tooth sectioning was discussed as well but would not be feasible because most of the root structure is shared. Some difficulty was encountered during accessing the distal part of the fused root due to the presence of inclination and this was achieved by modifying the long access of the bur.

In summary, the prevalence of such anomaly was not studied enough, and further investigation is needed to understand the effect of the ethnic factor or any other contributary factors. The current case demonstrates the importance of professional consultation at early stage and the role of CBCT in treatment planning. 


\section{REFERENCES}

1. Woelfel JB, Scheid RC. Dental anatomy: its relevance to dentistry. Williams \& wilkins; 1997.

2. Endodontists AA of. Glossary of endodontic terms [Internet]. American Association of Endodontists; 2015. Available from: https://www.aae.org/specialty/clinicalresources/glossary-endodontic-terms/

3. Oliván Rosas G, López Jiménez J, Giménez Prats MJ, Piqueras Hernández M. Consideraciones y diferencias en el tratamiento de un diente fusionado / Considerations and differences in the treatment of a fused tooth. Med Oral, Patol Oral y Cirugía Bucal (Ed impresa). 2004;9(3):224-8.
4. Steinbock N, Wigler R, Kaufman AY, Lin S, Abu-El Naaj I, Aizenbud D. Fusion of central incisors with supernumerary teeth: A 10-year follow-up of multidisciplinary treatment. J Endod. 2014;

5. Gautam G, Kumari VS, Jayashankar CM, Hadge P, Garg G. Interdisciplinary management of dental anomalies: fusion and supernumerary teeth. Orthodontics (Chic). 2011;

6. Mader CL. Fusion of teeth. J Am Dent Assoc [Internet]. 1979 Jan [cited 2020 Mar 21];98(1):62-4. Available from: https:// linkinghub.elsevier.com/retrieve/pii/S0002817779810272

7. Fayad MI, Nair M, Levin MD, Benavides E, Rubinstein RA, Barghan S, et al. AAE and AAOMR Joint Position Statement. Oral Surg Oral Med Oral Pathol Oral Radiol. 2015; 